\section{Traitement de l'hépatite C}

Nathalie Boyer, Patrick Marcellin

> Depuis la découverte du virus de l'hépatite C en 1989, le traitement de l'hépatite $C$ a considérablement progressé. Avec l'interféron $\alpha$ en monothérapie, le taux de réponses virologiques prolongées est inférieur à $20 \%$. Par la suite, l'utilisation d'une bithérapie associant l'interféron $\alpha$ et la ribavirine a permis d'obtenir $40 \%$ de réponses prolongées. Plus récemment, il a été démontré que les interférons pégylés sont globalement deux fois plus efficaces que l'interféron standard. La bithérapie associant les interférons pégylés et la ribavirine donne environ $55 \%$ de réponse virologique prolongée. Avec le recul, il apparaît que la réponse virologique prolongée est associée à un bénéfice histologique et probablement à une diminution du risque de cirrhose et de carcinome hépatocellulaire. Afin d'améliorer l'efficacité du traitement, plusieurs stratégies thérapeutiques sont envisagées : trithérapie (interféron pégylé + ribavirine + amantadine), cytokines, inhibiteurs enzymatiques, oligonucléotides anti-sens, ribozymes ou vaccin thérapeutique. <

Depuis la découverte du virus de l'hépatite C (VHC) en 1989, l'efficacité du traitement de l'hépatite chronique C n'a cessé de progresser (Figure 1). En 1989, la monothérapie avec l'interféron $\alpha$ (IFN) standard permettait d'obtenir moins de $10 \%$ de réponse virologique prolongée (négativation de l'ARN viral persistant 6 mois après l'arrêt du traitement). Avec la bithérapie associant l'IFN et la ribavirine, le taux de réponse prolongée était d'environ $40 \%$ en 1998. Les nouveaux IFN pégylés conjugués à du polyéthylène glycol (IFN-PEG), utilisés en monothérapie, sont globalement deux fois plus efficaces que les IFN standards et, récemment, deux grands essais contrôlés ont montré que la nouvelle bithérapie associant un IFN-PEG et la ribavirine permet d'obtenir environ $55 \%$ de réponses prolongées. Chez les patients atteints d'hépatite chronique C, l'objectif du traitement est d'arrêter la réplication virale pour diminuer

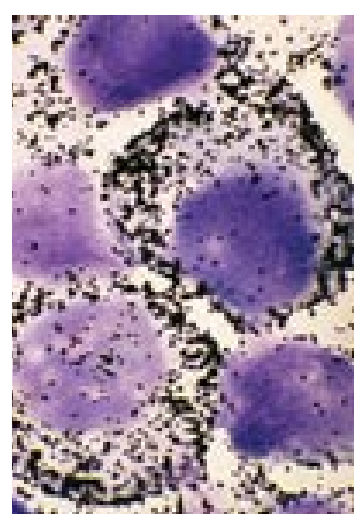

les lésions hépatiques de nécrose et d'inflammation afin de prévenir l'évolution de la fibrose vers la cirrhose et le carcinome hépatocellulaire.

\section{Interféron et ribavirine : des mécanismes d'action mal connus}

\section{L' interféron $\alpha$}

L'effet antiviral de I'IFN $\alpha$ dans l'hépatite chronique C est bien démontré avec une rapide diminution du taux d'ARN VHC sérique suivie d'une diminution du taux sérique des transaminases (ALAT) [1]. Les IFN sont des cytokines endogènes sécrétées en réponse à de nombreux stimulus - en particulier les infections virales - et dont l'activité antivirale est à l'origine de leur découverte. Il en existe environ 13 sous-types doués de nombreuses activités biologiques dont l'inhibition de la réplication virale, l'inhibition de la multiplication cellulaire, l'induction de l'apoptose ainsi que la modulation de la différenciation et de la réponse immunitaire. L'IFN, produit par toutes les cellules en réponse aux virus ou à I'ARN double-brin, active une cascade de signalisation qui aboutit à l'induction de nombreuses protéines dont 
le rôle, pour la plupart, n'est pas encore élucidé. Les mieux connues sont la protéine kinase dépendante de I'ARN double brin (PKR), le système 2'5'oligoadénylate synthétase /RNase $L$, et certaines formes de protéines Mx. L'activation des deux premières voies enzymatiques entraîne une inhibition non spécifique de la réplication virale, en inhibant la synthèse des protéines. En outre, I'IFN stimule la synthèse et la présentation des protéines du système majeur d'histocompatibilité de classe I et II qui sont impliquées dans la présentation des épitopes viraux aux lymphocytes $C D 4^{+}$et $C D 8^{+}$, ce qui stimule la prolifération des cellules $T$ pendant l'activation de la réponse immunitaire.

Dans l'hépatite chronique C, il a été démontré que l'IFN a essentiellement quatre fonctions [2] : diminution de la réplication virale; induction d'un état antiviral dans les cellules non infectées; augmentation de la lyse des cellules infectées; inhibition de la fibrogenèse hépatique. La diminution de la réplication virale apparaît dans les 6 à 8 heures qui suivent l'injection d'interféron, un délai qui correspond à l'activation des mécanismes antiviraux des hépatocytes. L'induction d'un état antiviral dans les hépatocytes non infectés prévient leur infection et donc la diffusion de l'infection. L'augmentation de la lyse des hépatocytes infectés résulte d'une augmentation de l'expression des complexes formés des peptides viraux et des molécules du système majeur d'histocompatibilité de la membrane cellulaire, et de la stimulation de l'activité des cellules NK (natural killer) et de l'activité CTL (lymphocytes T cytotoxiques). De plus, il a été montré que l'IFN réduit non seulement les lésions histologiques de nécrose et d'inflammation (activité histologique), mais aussi celles de fibrose. Cette amélioration histologique peut être observée chez des patients n'ayant pas de réponse virologique. L'effet anti-fibrosant de l'IFN pourrait être lié à l'inhibition du TGF $\beta$ (transforming growth factor $\beta$ ). Certaines études suggèrent que I'IFN pourrait diminuer le risque de développement d'un carcinome hépatocellulaire mais cela reste controversé.

Des études récentes montrent que la cinétique de diminution de la charge virale sous traitement par IFN est biphasique (Figure 2) [3]. Pendant la première phase, qui dure environ 24 heures, la charge virale diminue rapidement, une diminution dont l'intensité est corrélée à la dose d'IFN administrée et serait due à l'effet antiviral direct de cette cytokine. Dans la seconde phase, la diminution de la charge virale est plus lente, elle s'étend sur plusieurs semaines voire plusieurs mois et serait en relation avec la clairance des cellules infectées. Cette diminution, qui dépend peu de la dose administrée, semble être essentiellement influencée par la qualité de la réponse immunitaire. Ainsi, si la première phase de décroissance de la charge virale est observée chez pratiquement tous les patients traités, la seconde ne l'est pas chez les patients non répondeurs.

\section{La ribavirine}

La ribavirine est un analogue nucléosidique de la guanosine qui a été découvert en 1972 et possède un large spectre d'action antivirale (myxovirus, virus respiratoire syncytial, flavivirus...). Dans les études de phase II et III, il a été observé que 30 à $50 \%$ des patients atteints d'hépatite chronique $C$ traités par la ribavirine en monothérapie avaient une normalisation des transaminases mais sans diminution significative de la charge virale. Cela suggérait que la ribavirine agit davantage par des mécanismes immunomodulateurs que par des effets directs antiviraux. Curieusement, l'association de la ribavirine à I'IFN augmente nettement l'efficacité du traitement en augmentant la proportion de patients ayant une réponse virologique.

Les mécanismes d'action de la ribavirine sur le VHC restent inconnus, les études expérimentales étant jusqu'à présent limitées par l'absence de système efficace de réplication du VHC en culture cellulaire et de modèle animal facilement disponible. C'est pourquoi les données disponibles ont été principalement obtenues à partir de systèmes de culture de virus apparentés au VHC, comme le BVD (bovine viral diarrhea virus) ou le poliovirus. La ribavirine, en inhibant l'inosine monophosphate déshydrogénase, vide le

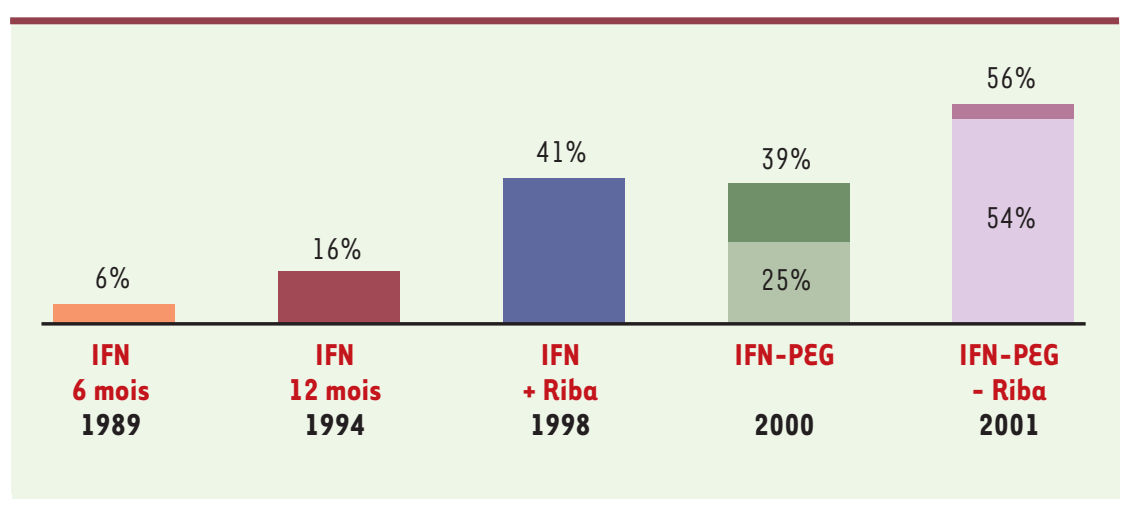

Figure 1. Évolution de l'efficacité du traitement de l'hépatite chronique C. Depuis 1989, le taux de réponses virologiques prolongées a augmenté de $6 \%$ avec l'interféron standard utilisé en monothérapie pendant 6 mois, à $16 \%$ quand le traitement est prolongé pendant 12 mois, puis à $41 \%$ en association avec la ribavirine. Les interférons pégylés sont globalement 2 fois plus efficaces que les interférons standards avec respectivement $25 \%$ et $39 \%$ de réponse virologique prolongée avec l'interféron pégylé $\alpha-2 b$ et l'interféron pégylé $\alpha-2 a$. La bi-thérapie associant les interférons pégylés $\alpha-2 b$ et $\alpha-2 a$ à la ribavirine donne respectivement $54 \%$ et $56 \%$ de réponses virologiques prolongées. 
stock intracellulaire de nucléotides (GTP et dGTP), ce qui entraîne une diminution de la réplication du génome viral. Elle inhibe aussi l'ARN polymérase de certains virus et la synthèse protéique cellulaire, ce qui peut affecter la synthèse des protéines virales. Des modifications du cycle cellulaire, en particulier un retard de la progression vers la phase $S$, ont été mises en évidence et pourraient retarder la réplication virale. Enfin, il a été récemment montré que la ribavirine avait un effet mutagène sur le génome du poliovirus, responsable d'une diminution de son pouvoir infectieux [4].
En outre, il semble que la ribavirine ait un effet immuno-modulateur, en favorisant la réponse T auxiliaire de type 1 (Thl) aux dépens de la réponse Th2 [5]. In vivo, chez la souris, la ribavirine favorise en effet la réponse Thl, en induisant notamment la production d'interleukine-12 (IL-12). Des études réalisées in vitro sur des cultures de lymphocytes T humains de sujets sains mettent en évidence un effet stimulateur de la ribavirine sur la réponse Thl en augmentant la production d'IL-2, d'IFN $\gamma$ et de TNF $\alpha$ tout en réprimant la réponse cytokine de type Th2 (IL-4, IL-5, IL10). Chez les malades traités par l'association ribavirine-IFN, il a été observé une diminution de la production d'IL-10 par les lymphocytes en culture par rapport aux malades traités par l'IFN seul, ce qui suggère une diminution de la réponse de type Th2.

\section{Le traitement de I'infection par le VHC}

L'efficacité du traitement est définie par l'acquisition d'une réponse virologique prolongée, c'est-àdire une charge virale négative persistant six mois après l'arrêt du traitement. Il paraît important de souligner que la notion de charge virale négative repose sur l'absence de détection de I'ARN viral dans le sérum des patients et est donc dépendante de la sensibilité des techniques utilisées pour cette détection. Les tests actuellement utilisés (PCR ou TMA, transcription mediated amplification) ont une limite de détection de l'ordre de 50 à 100 copies par ml. La négativation de la charge virale pendant le traitement est nécessaire pour obtenir une réponse prolongée qui est généralement associée à une

Figure 2. Cinétique de diminution de la charge virale avec un traitement par l'interféron. La cinétique de diminution de la charge virale sous traitement par IFN est biphasique. L'administration d'IFN $\alpha$ entraine dans une première phase une diminution rapide, dès le premier jour, de l'ARN VHC suivie d'une deuxième phase de diminution plus lente. La rapidité de diminution de l'ARN VHC sérique dépend de la dose d'IFN. Cinétique de l'ARN VHC sérique pendant les 2 premiers jours du traitement (à gauche) et pendant les 14 premiers jours du traitement (à droite) chez des malades recevant (de haut en bas) une dose de 2 MU, 5 MU, et 10 MU d'IFN $\alpha$. 
réponse biochimique prolongée (normalisation des transaminases ALAT) [6]. Dans plus de $95 \%$ des cas, cette réponse est durable, sans rechute tardive, et s'accompagne d'une amélioration progressive des lésions histologiques hépatiques qui peuvent parfois totalement disparaître $[6,7]$. Bien que certaines études aient montré que l'ARN viral était indétectable (par PCR) dans le foie de patients présentant une réponse virologique prolongée 2 à 5 ans après l'arrêt du traitement [6], l'éradication de l'infection par le VHC n'est pas entièrement prouvée et le bénéfice sur la survie est certes probable mais n'a pas été clairement démontré.

De nombreux essais cliniques ont été effectués, visant à évaluer l'efficacité des différents traitements, leurs indications, les posologies et la durée de traitement nécessaires. Leurs résultats sont parfois contradictoires, en fonction du caractère contrôlé ou non des études, des critères d'inclusion des patients, du nombre de patients inclus... Les études contrôlées ont toutefois permis de préciser les schémas thérapeutiques efficaces qui font l'objet de recommandations au cours des Conférences de Consensus sur l'hépatite C.

\section{Une efficacité croissante des thérapeutiques}

Depuis la première étude effectuée en 1986 [8], l'efficacité du traitement par l'IFN chez une minorité de patients atteints d'hépatite chronique $\mathrm{C}$ a été claire- ment démontrée. Les nombreuses études contrôlées ont permis d'établir un schéma thérapeutique de référence de 3 millions d'unités (MU), 3 fois par semaine pendant 12 mois $[1,9]$ (Tableau I). Ce schéma ne permet cependant d'obtenir une réponse prolongée que dans moins de $20 \%$ des cas. L'utilisation de doses plus élevées d'IFN ou l'allongement de la durée du traitement n'a pas permis d'améliorer l'efficacité thérapeutique et se sont accompagnés d'une plus mauvaise tolérance $[9,10]$.

Une première amélioration importante est apparue en 1998 avec l'utilisation d'une bithérapie associant la ribavirine à I'IFN. Deux larges études contrôlées ont en effet confirmé que cette bithérapie conduite pendant 24 à 48 semaines permettait l'obtention d'un taux de réponses prolongées nettement supérieur à celui obtenu avec I'IFN seul [11, 12] (Tableau I). Ces résultats ont conduit à considérer la bi-thérapie comme le traitement de référence de l'hépatite chronique C, comme l'a recommandé la conférence de Consensus Internationale sur l'hépatite C qui s'est tenue à Paris en 1999 [13].

Plus récemment, le remplacement de I'IFN standard par de I'IFN-PEG a permis d'accroître encore l'efficacité du traitement. Cette modification chimique diminue l'élimination rénale de l'IFN et augmente donc de façon importante sa demi-vie. Cela permet d'obtenir une concentration plasmatique d'IFN plus stable et prolongée pendant une semaine (Figure 3) [14]. Par ailleurs, la

\begin{tabular}{|c|c|c|c|c|}
\hline Protocole & Dose & $\begin{array}{c}\text { Durée } \\
\text { (semaines) }\end{array}$ & $\begin{array}{c}\text { Taux de réponses } \\
\text { virologiques }\end{array}$ & Réf \\
\hline IFN $\alpha-2 b$ & $3 \mathrm{MU}$ & 24 & $6 \%$ & \multirow{4}{*}{$\begin{array}{l}\text { [11] } \\
{[12]}\end{array}$} \\
\hline IFN $\alpha-2 b+$ ribavirine & $3 \mathrm{MU}+1000-1200 \mathrm{mg}$ & 24 & $33 \%$ & \\
\hline IFN $\alpha-2 b$ & $3 \mathrm{MU}$ & 48 & $16 \%$ & \\
\hline IFN $\alpha-2 b+$ ribavirine & $3 \mathrm{MU}+1000-1200 \mathrm{mg}$ & 48 & $41 \%$ & \\
\hline IFN $\alpha-2 a$ & $6 \mathrm{MU}$ puis $3 \mathrm{MU}$ & 48 & $19 \%$ & \multirow{2}{*}{ [15] } \\
\hline IFN-PEG $\alpha-2 a$ & $180 \mu \mathrm{g} / \mathrm{sem}$ & 48 & $39 \%$ & \\
\hline IFN $\alpha-2 b$ & $3 \mathrm{MU}$ & 48 & $12 \%$ & \multirow{2}{*}{ [16] } \\
\hline IFN-PEG $\alpha-2 b$ & $1 \mu \mathrm{g} / \mathrm{sem}$ & 48 & $25 \%$ & \\
\hline IFN $\alpha-2 b+$ ribavirine & $3 \mathrm{MU}+1000-1200 \mathrm{mg}$ & 48 & $47 \%$ & \multirow{3}{*}[17]{} \\
\hline IFN-PEG $\alpha-2 b+$ ribavirine & 1,5 puis $1 \mu \mathrm{g} / \mathrm{s}+1000-1200 \mathrm{mg}$ & 48 & $47 \%$ & \\
\hline IFN-PEG $\alpha-2 b+$ ribavirine & $1,5 \mu \mathrm{g} / \mathrm{s}+800 \mathrm{mg}$ & 48 & $54 \%$ & \\
\hline IFN $\alpha-2 b+$ ribavirine & $3 \mathrm{MU}+1000-1200 \mathrm{mg}$ & 48 & $45 \%$ & \multirow{3}{*}[18]{} \\
\hline IFN-PEG $\alpha-2 a$ & $180 \mu \mathrm{g} / \mathrm{sem}$ & 48 & $30 \%$ & \\
\hline IFN-PEG $\alpha-2 a+$ ribavirine & $180 \mu \mathrm{g} / \mathrm{sem}+1000-1200 \mathrm{mg}$ & 48 & $56 \%$ & \\
\hline
\end{tabular}

Tableau I. Principaux essais contrôlés randomisés de la bi-thérapie IFN standard-ribavirine et de la bi-thérapie IFN pégylé-ribavirine chez les malades atteints d'hépatite chronique C. Les doses d'IFN standard sont administrées trois fois par semaine et celles d'IFN-PEG une fois par semaine. La posologie indiquée de ribavirine correspond à la prise quotidienne par voie orale. 
pégylation diminue l'immunogénicité de la protéine (diminution de la production d'anticorps anti-IFN). En outre, l'amélioration de la pharmacocinétique permet de réduire le nombre d'injections de trois à une, ce qui constitue indéniablement une amélioration du confort du malade.

Deux IFN-PEG, qui diffèrent par la qualité et la quantité de PEG conjugué à l'IFN, ont été évalués chez des patients atteints d'hépatite chronique $C$ : un PEG linéaire de $12 \mathrm{kDa}$ pour l'IFN $\alpha-2 \mathrm{~b}$ et un PEG ramifié de $40 \mathrm{kDa}$ pour l'IFN $\alpha-2 a$. Dans les deux cas, I'IFN-PEG se révèle globalement deux fois plus efficace que l'IFN correspondant non conjugué $[15,16]$ (Tableau I). Et surtout, leur association à la ribavirine a permis d'accroître encore l'efficacité de la bithérapie à un taux de $55 \%$ de réponses prolongées, ce qui constitue la meilleure efficacité obtenue dans le traitement de l'hépatite C $[17,18]$.

\section{De nombreux cas particuliers}

Dans les grands essais cliniques précédemment décrits, les patients ont été sélectionnés selon des critères
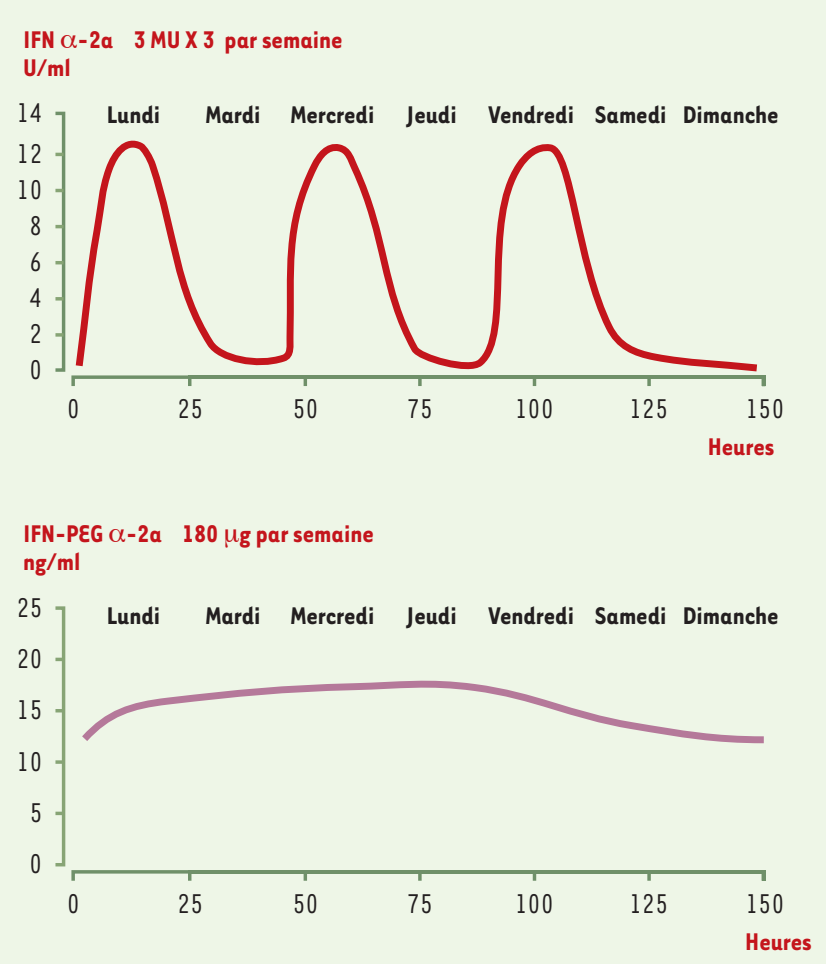

Figure 3. Pharmacocinétique de l'IFN standard et de l'IFN pégylé. La concentration plasmatique d'interféron standard (ici interféron $\alpha-2 a$ ) administré par voie sous-cutanée 3 fois par semaine est très variable avec des pics suivis de concentrations très faibles. A l'inverse, la concentration obtenue avec l'interféron pégylé (ici interféron pégylé $\alpha-2 a$ ) administré par voie sous-cutanée 1 fois par semaine est plus stable et plus prolongée. stricts. C'est pourquoi l'efficacité des traitements est encore mal connue dans un certain nombre de groupes de patients, importants par leur nombre ou par la sévérité de la maladie, et pour lesquels les études contrôlées ne sont pas toujours disponibles.

Un problème important est posé par les patients non répondeurs à un premier traitement par I'IFN seul. Une nouvelle monothérapie est inefficace [19] et la bi-thérapie l'est rarement, avec seulement 5 à $20 \%$ de réponses prolongées [20]. Une étude récente suggère qu'une trithérapie combinant l'IFN, la ribavirine et l'amantadine pourrait être efficace chez ces malades [21]. L'amantadine est un agent antiviral hydrosoluble actif sur le virus Influenza $A$. Cet agent antiviral bloque la protéine de la matrice membranaire (M2) qui fonctionne comme un canal ionique nécessaire à l'internalisation du virus par endocytose [22]. Mais ces résultats doivent être confirmés par de plus larges études contrôlées en cours de réalisation.

Un autre cas particulier est celui des patients au stade de cirrhose, pour lesquels l'efficacité du traitement est moindre : moins de $5 \%$ de réponses virologiques prolongées avec l'IFN seul [23], et $20 \%$ à $30 \%$ avec la bithérapie [24]. Certaines études ont suggéré que le traitement par l'IFN pouvait toutefois diminuer le risque de complications de la cirrhose et, en particulier, l'incidence du carcinome hépatocellulaire [25]. Mais cette hypothèse reste controversée, d'autres études n'ayant pas retrouvé de différence entre les patients traités et non traités [26]. Si le bénéfice est probable chez les patients répondeurs, le bénéfice potentiel n'est pas démontré chez les non répondeurs [27]. Des études prospectives contrôlées sont en cours pour évaluer le bénéfice à long terme du traitement chez les patients avec cirrhose.

Environ $25 \%$ des malades atteints d'hépatite chronique $\mathrm{C}$ ont une forme peu évolutive de la maladie avec des transaminases normales et des lésions hépatiques généralement minimes [28]. Dans ce cas, le taux de réponse à la monothérapie ne semble pas différent de celui des autres patients et les résultats de la bithérapie sont en cours d'évaluation. Ainsi, compte tenu du caractère peu évolutif de cette forme de la maladie, le traitement n'était jusqu'à présent pas recommandé [29]. 
$(\rightarrow) \mathrm{m} / \mathrm{s}$

1999, nº10,

p. 1191
Enfin, quand le diagnostic d'infection par le VHC est porté au stade de l'hépatite aiguë, il est clairement établi que le traitement (seule la monothérapie a été évaluée) diminue le risque d'évolution vers la chronicité [30] (environ $50 \%$ au lieu de $80 \%)$. Un taux de réponses particulièrement élevé (98\%) semble même obtenu avec un schéma thérapeutique plus intensif [31].

Chez les patients atteints d'hépatite chronique ayant une co-infection VIH, l'ensemble des études suggère que le traitement par IFN seul est moins efficace qu'en l'absence de co-infection VIH : de 0 à $22 \%$ de réponses chez les patients positifs pour le VIH contre 19 à $30 \%$ chez les patients non infectés par le VIH $[32,33]$. Deux études pilotes utilisant la bi-thérapie [34, 35] montrent des taux de réponses qui semblent inférieurs à ceux habituellement observés chez les malades non infectés par le VIH. Des complications graves liées à une possible toxicité mitochondriale de la ribavirine ont été décrites récemment. Elles pourraient être favorisées par l'existence d'interactions entre la ribavirine et certains antirétroviraux (zidovudine et stavudine) $(\rightarrow)$ [32].

\section{Quand la transplantation hépatique \\ devient le seul recours}

L'hépatite chronique C est la première cause (environ $30 \%$ ) de transplantation hépatique aux États-Unis et en Europe. La transplantation peut être justifiée par l'existence d'une cirrhose compliquée, d'un carcinome hépatocellulaire ou l'association des deux. Les résultats de la transplantation sont essentiellement limités par la récidive quasi constante de l'infection par le VHC sur le greffon, qui se traduit par la réapparition, en moyenne deux semaines après la transplantation, de l'ARN viral dans le sérum [36]. Si, jusqu'à présent, la survie à cinq ans était voisine de celle des patients ayant bénéficié d'une transplantation pour d'autres étiologies, on observe dans des études récentes une évolution plus sévère (dont les raisons ne sont pas comprises) avec une incidence élevée de cirrhose du greffon. La tendance actuelle est de réduire le plus rapidement possible les doses d'immunosuppresseurs qui pourraient être un des principaux facteurs aggravant l'évolution de l'hépatite chronique $C$ du greffon. Des études pilotes suggèrent que la bithérapie standard pourrait être efficace mais elle est moins bien tolérée dans cette population [37].

\section{Facteurs prédictifs de la réponse au traitement}

La probabilité d'obtenir une réponse prolongée au traitement dépend surtout du génotype et de la charge virale. L'âge jeune, le sexe féminin et une fibrose absente ou faible, sont également associés, à un moindre degré, à une bonne réponse. Malheureu- sement, le génotype l (la ou lb), qui est associé à une mauvaise réponse au traitement, est le génotype le plus fréquent en Europe et aux États-Unis, présent dans 60 à $80 \%$ des cas en fonction des pays. En France, ce génotype est présent dans $57 \%$ des cas, tandis que les génotypes 2 et 3 , qui sont associés à une bonne réponse au traitement, ne représentent respectivement que $11 \%$ et $22 \%$ des cas [38].

La monothérapie par l'IFN induit une réponse prolongée dans $30 \%$ des cas pour les génotypes 2 ou 3, mais seulement dans $5 \%$ pour le génotype l (la ou lb) [39]. Avec la bi-thérapie standard, le taux de réponse prolongée est doublé, atteignant $60 \%$ pour les génotypes 2 ou 3, quelle que soit la charge virale, et augmente à $35 \%$ pour le génotype 1 associé à une faible charge virale (définie par moins de 2 millions de copies d'ARN viral/ml) $[11,12]$. La durée du traitement (6 ou 12 mois) n'a d'influence sur la réponse prolongée que dans le cas du génotype 1 associé à une forte charge virale [13].

Avec la bi-thérapie IFN-PEG, les résultats sont discordants. La bi-thérapie est significativement plus efficace avec I'IFN-PEG $\alpha-2 b$ pour le génotype 1 mais pas pour les génotypes 2 ou 3 [17]. La bi-thérapie avec I'IFN-PEG $\alpha-2 a$ est significativement plus efficace pour tous les génotypes [18].

La négativation précoce de l'ARN VHC sérique pendant le traitement par l'IFN est le meilleur facteur prédictif de réponse prolongée [3]. Ainsi, il semble que le traitement puisse être interrompu si l'ARN du VHC sérique reste détectable après 3 mois de traitement et si la charge virale a diminué de moins de 2 unités logarithmiques dans la mesure où la probabilité d'une réponse prolongée est, dans ce cas, de l'ordre de $3 \%$ [18]. Ce critère est retrouvé dans toutes les études évaluant la bithérapie.

\section{Des effets secondaires}

Le principal effet secondaire de l'IFN est le syndrome pseudo-grippal. Habituellement modéré et bien contrôlé par le paracétamol [40], il est parfois sévère nécessitant alors une diminution de la posologie. Plus rarement, il peut conduire à un arrêt du traitement, tout comme l'apparition d'un syndrome dépressif sévère ou d'une dysthyroïdie. Toutes causes confondues, l'arrêt du traitement n'est nécessaire que dans moins de $10 \%$ des cas.

La tolérance de I'IFN-PEG en monothérapie est globalement comparable à celle de l'IFN standard [15, 16]. Les effets secondaires sévères, en particulier psychiatriques, ne sont pas plus fréquents. Le syndrome grippal et les signes cutanés inflammatoires au point d'injection sont un peu plus fréquents, ainsi que la neutropénie. La posologie a été diminuée un peu plus souvent 
avec l'IFN-PEG qu'avec l'IFN essentiellement à cause de l'apparition d'une neutropénie. La tolérance de la bithérapie lorsque I'IFN-PEG $\alpha$-2b est utilisé à la dose de $1,5 \mu \mathrm{g} / \mathrm{kg}$ semble peu différente de celle de la bi-thérapie standard, avec une diminution de dose étant un peu plus fréquente ( $42 \%$ des cas contre $34 \%$ ), mais sans augmentation de la proportion de cas où l'arrêt du traitement a été nécessaire (14\% des cas contre $13 \%$ ) [17].

\section{Mécanismes de résistance au traitement}

Malgré l'amélioration relativement rapide de l'efficacité du traitement au cours des dernières années, avec actuellement environ $55 \%$ de réponses prolongées avec la bithérapie associant I'IFN-PEG et la ribavirine, près de la moitié des patients ne répondent pas, ou seulement partiellement, au traitement. Si les mécanismes de résistance ne sont pas bien connus, il est clair que des facteurs virologiques sont impliqués.

Tout d'abord, le VHC se réplique très rapidement. On estime qu'environ $10^{12}$ virions sont produits chaque jour [3]. La demi-vie des virions est courte, de l'ordre de 3 heures. Ainsi, le taux élevé de réplication virale est probablement une des premières causes d'échec du traitement. On observe d'ailleurs que la réponse au traitement est corrélée à la charge virale pré-thérapeutique.

L'intensité de la réplication virale favorise l'accumulation de mutations au niveau du génome viral. Elles sont à l'origine de ce que l'on appelle la quasi-espèce virale, c'est-à-dire la coexistence de variants viraux génétiquement distincts mais apparentés, car tous dérivent du même inoculum. Cette variabilité du VHC explique, au moins en partie, les phénomènes de résistance au traitement du fait de la pression de sélection positive qui favorise la survie des mutants les mieux adaptés à la pression immunitaire induite par l'IFN [41]. II a d'ailleurs été observé que la réponse au traitement était inversement corrélée à l'hétérogénéité de la quasi-espèce (nombre de variants) [42].

Si les mécanismes précis de résistance à l'IFN de certaines souches virales demeurent actuellement inconnus, des études suggèrent qu'une région spécifique $\mathrm{du}$ génome viral localisée dans la région codant pour la protéine non structurale NS5A est associée à une résistance à I'IFN (région déterminant la sensibilité à l'interféron ou ISDR) [43]. L'hypothèse proposée est que la protéine NS5A a la capacité d'inactiver la protéine kinase PKR. La PKR est une des protéines induites par I'IFN ; elle phosphoryle et inactive le facteur d'initiation de la translation, elF2, ce qui inhibe la synthèse des protéines et empêche la réplication virale. Avec les souches résistantes à l'IFN, la liaison de NS5A à la PKR inactiverait la protéine kinase tandis qu'avec les souches sensibles à I'IFN, la protéine NS5A n'aurait pas la capacité de se lier à la PKR, qui pourrait alors bloquer la réplication virale. C'est la première démonstration d'un mécanisme spécifique par lequel le VHC aurait la capacité de résister à l'interféron. Cependant, plusieurs études analysant précisément la région ISDR ont donné des résultats contradictoires suggérant que d'autres régions du génome pourraient être impliquées. D’autres études moléculaires sur la résistance du VHC sont cruciales pour mieux comprendre les stratégies moléculaires développées par le VHC pour échapper au traitement. II est également possible que la charge virale intra-hépatique, qui reflète mieux l'intensité de la réplication virale, et le nombre de cellules hépatiques infectées, jouent un rôle important dans la résistance au traitement [44].

\section{Perspectives}

En dépit des progrès importants des recherches sur l'hépatite $C$ au cours des dix dernières années, de nombreuses questions restent posées qui concernent aussi bien la compréhension de la pathogénie de la maladie que la prise en charge des malades.

Le premier problème est de mieux connaître l'histoire naturelle de l'infection par le VHC et de préciser les facteurs pronostiques. Pour cela, des études comprenant de larges cohortes sont nécessaires. Idéalement, des procédés moins invasifs que la biopsie hépatique, comme les marqueurs sanguins de fibrose $(\rightarrow)$, devraient être développés afin d'apprécier la sévérité de l'atteinte hépatique. L'influence des facteurs tels que l'âge, le sexe et l'alcool doivent être mieux compris et d'autres facteurs potentiels importants, encore inconnus, restent à déterminer. L'un des problèmes les plus urgents est de comprendre les mécanismes favorisant la fibrogenèse et la carcinogenèse associées à l'hépatite chronique $\mathrm{C}$.

Le second problème est d'améliorer l'efficacité des traitements. Bien que l'association de la ribavirine à l'IFNPEG soit sans aucun doute un progrès important, les résultats restent insatisfaisants avec environ $45 \%$ de patients non répondeurs. Une meilleure utilisation des drogues actuellement disponibles ne permettra probablement pas d'obtenir des résultats nettement supérieurs. Le développement de nouvelles molécules s'avère donc nécessaire, comme les inhibiteurs des enzymes virales (protéases, hélicases et polymérases). La résolution récente des structures tridimensionnelles des $\rightarrow$ m/s 2002, n०3, p. 353 
protéases [45] et des hélicases [46] virales représente une avancée importante car elle permet de développer des inhibiteurs spécifiques de ces enzymes.

Une approche thérapeutique innovante repose sur l'utilisation d'oligonucléotides anti-sens, conçus pour s'hybrider spécifiquement aux ARN viraux et inhiber la réplication du VHC. Une autre approche moléculaire est l'utilisation des ribozymes (acides ribonucléiques ayant une activité enzymatique) [47].

Les cytokines et les modulateurs des cytokines méritent aussi d'être évalués. Parmi eux, l'IL-12 a des propriétés immunomodulatrices intéressantes : elle induit la différenciation des cellules CD4 en cellules effectrices Thl et augmente la réponse immune Thl induite par les antigènes. Cette cytokine est actuellement en cours d'évaluation clinique. L'IL-10, qui a des effets anti-inflammatoires, anti-fibrosants et auto-immuns, pourrait également être intéressante.

Finalement, le principal défi est le développement de vaccins, qui se heurte à plusieurs problèmes scientifiques et pratiques majeurs [48] : (1) la grande variabilité des protéines virales; (2) l'absence, en dehors du chimpanzé, de modèle animal d'infection par le VHC ; (3) l'absence de système de réplication efficace in vitro. Le développement de modèles animaux et de culture in vitro représente actuellement un enjeu majeur. Il a été observé chez le chimpanzé que des protéines d'enveloppe recombinantes peuvent entraîner une réponse anticorps et une réponse des cellules $T$ CD4 ${ }^{+}$ [49]. Mais les candidats pour les vaccins protecteurs semblent être encore lointains et la mise au point de vaccins thérapeutiques paraisse à court terme plus réaliste.

En conclusion, l'hépatite $C$ est un problème majeur de santé publique. Une augmentation de l'incidence des cirrhoses et des carcinomes hépatocellulaires liés au VHC est attendue dans les dix prochaines années. Un dépistage à large échelle et l'amélioration des traitements sont nécessaires pour ralentir ou arrêter la progression de la maladie hépatique chez les sujets infectés. Cela signifie que des efforts majeurs sont nécessaires pour améliorer l'efficacité et réduire le coût des traitements, afin de permettre une meilleure prise en charge du plus grand nombre de patients dans le monde. $\diamond$

\section{SUMMARY}

Treatment of hepatitis $C$

Since the discovering of the hepatitis C virus in 1989, the treatment of hepatitis C has considerably improved. Initially, with interferon alpha used as a single drug, the sustained virological response rate was below $20 \%$. Then, with the use of combination therapy of interferon alpha with ribavirin, the response rate increased to $41 \%$. More recently, pegylated interferons were shown to be twice more effective than standard interferons. The combination of pegylated interferons with ribavirin give a response rate of about $55 \%$. The long-term follow-up studies showed that sustained virological response is generally associated with clinical and histological improvement and probably with a decreased risk of development of cirrhosis and hepatocellular carcinoma. The indication of therapy is mainly based on the results of the liver biopsy which is the best way to assess the prognosis of the liver disease. Therefore, treatment is indicated in patients with moderate or severe necroinflammation or fibrosis. The tolerability of combination therapy is relatively poor with a frequent flulike syndrom and an impaired quality of life. The most frequent adverse events are depression, thyroid dysfunction, neutropenia and anemia which need reduction of dosage in about $40 \%$ and interruption of treatment in about $10 \%$ of treated patients. The chances to obtain a sustained virological response depends essentially on the viral genotype and the viral load. To further improve the efficacy of therapy, different new drugs are under investigation (amantadine, cytokines). These drugs may be candidates for new combinations. In addition, intensive research is currently done for the development of inhibitors of viral enzymes (helicase, proteases or polymerase) and anti-sense oligonucleotides, ribozymes and therapeutic vaccine. $\diamond$

\section{RÉFÉRENCES}

1. Hoofnagle JH, Di Bisceglie AM. The treatment of chronic viral hepatitis. N Engl J Med 1997 ; 226 : 347-56.

2. Thomas HC, Törok ME, Forton DM, et al. Possible mechanisms of action and reasons for failure of antiviral therapy in chronic hepatitis C. J Hepatol 1999 32 (suppl 1) : 152-9.

3. Neumann AU, Lam NP, Dahari $\mathrm{H}$, et al. Hepatitis C viral dynamics in vivo and the antiviral efficacy of interferon- $\alpha$ therapy. Science 1998 ; 282 : 103-7.

4. Crotty S, Maag D, Arnold JJ, et al. The broad-spectrum antiviral ribonucleoside ribavirin is an RNA virus mutagen. Nat Med 2000 ; $6:$ 1375-9.

5. Hultgren C, Milich DR, Weiland 0 , et al. The antiviral compound ribavirin modulates the $T$ helper (Th) 1/Th2 subset balance in hepatitis $B$ and $C$ virus-specific immune responses. J Gen Virol 1998 ; 79 : 2381-91. 
6. Marcellin P, Boyer N, Gervais $A$, et al. Long-term histologic improvement and loss of detectable intrahepatic HCV RNA in patients with chronic hepatitis $C$ and sustained response to interferon- $a$ therapy. Ann Intern Med 1997 ; $127: 875-81$.

7. Duchatelle V, Marcellin P, Giostra $\varepsilon$, et al. Changes in liver fibrosis at the end of alpha interferon therapy and 6 to 18 months later in patients with chronic hepatitis $C$ : quantitative assement by a morphometric method. J Hepatol 1998 ; $29: 20-8$.

8. Hoofnagle JH, Mullen KD, Jones DB, et al. Treatment of chronic non-A, non-B hepatitis with recombinant human alpha interferon : a preliminary report. $N$ EnglJ Med 1986 ; 315 : 1575-8.

9. Marcellin P. Traitement de I'hépatite $C$ par I'interféron. Conférence de Consensus - Textes des experts. Comment traiter I'hépatite C ? Gastroenterol Clin Biol 1997 ; 20 : S81-S88.

10. Shiffman ML. Use of highdose interferon in the treatment of chronic hepatitis C. Sem Liver Dis. $1999 ; 19$ (Suppl 1) : 25-33.

11. Poynard T, Marcellin P, Lee SS, et al. Randomised trial of interferon $a 2 b$ plus ribavirin for 48 weeks or for 24 weeks versus interferon $\mathrm{a} 2 \mathrm{~b}$ plus placebo for 48 weeks for treatment of chronic infection with hepatitis $\mathrm{C}$ virus. Lancet 1998 ; 352 : 1426-32.

12. McHutchison JG, Gordon SC, Schiff $\varepsilon R$, et al. Interferon alfa- $2 b$ alone or in combination with ribavirin as initial treatment for chronic hepatitis C. N EnglJ Med 1998 ; 339 : 1485-92.
13. EASL International Consensus Conference on Hepatitis C. Consensus Statement. J Hepatol 1999 ; 30: 956-61

14. Boyer N, Marcellin P. L'interféron pegylé : nouveau progrès dans le traitement de l'hépatite chronique C. Gastroentérol Clin Biol 2000 ; 24 : 767-9.

15. Zeuzem S, Feinman SV, Rasenack J, et al. Peginterferon alfa-2a in patients with chronic hepatitis C. N Engl J Med 2000 ; 343 : 1666-72.

16. Lindsay KL, Trépo C, Heintges $T$, et al. A randomized, double-blind trial comparing pegylated interferon alfa-2b to interferon alfa-2b as initial treatment for chronic hepatitis C. Hepatology 2001 ; 34 : 395-403

17. Manns MP, McHutchison JG, Gordon $S$, et al. Peginterferon alfa-2b plus ribavirin compared with IFN-2b plus ribavirin for initial treatment of chronic hepatitis $C$ : a randomised trial. Lancet 2001 ; 358 : 958-65.

18. Fried MW, Shiffman ML, Reddy RK, et al. Pegylated (40 kDa) interferon alfa-2a

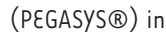
combination with ribavirin efficacy and safety results from a phase III, randomized, activelycontrolled, multicenter study. Gastroenteroly 2001 ; 120 : A55 (abstract)

19. Chow WC, Boyer N, Pouteau $M$, et al. Retreatment with interferon alfa of patients with chronic hepatitis C. Hepatology 1998 ; 27 : 1144-8.

20. Pol $S$, Couzigou $P$, Bourlière $M$, et al. A randomized trial of ribavirin and interferona vs. interferon-a alone in patients with chronic hepatitis C who were nonresponders to a previous treatment. J Hepatol 1999 ; $31: 1-7$.

21. Brillanti S, Levantesi F, Masi $L$, et al. Triple antiviral therapy as a new option for patients with interferon nonresponsive chronic hepatitis C. Hepatology $2000 ; 32$ : 630-4.

22. Wright M, main J, Thomas HC. Drug-resistant viral hepatitis. J Infect 2000 ; $41: 1-4$.

23. Valla $D$, Chevallier $M$, Marcellin $\mathrm{P}$, et al. Treatment of hepatitis C virus-related cirrhosis. $A$ randomized controlled trial of IFN-2b versus non treatment. Hepatology 1999 ; 29 : 1870-5.

24. Schalm SW, Weiland 0 , Hansen BE, et al. Interferon-ribavirin for chronic hepatitis C with and without cirrhosis : analysis of individual patient data of six controlled trials. Gastroenterology 1999 ; 117 : 408-13.

25. Nishiguchi $S$, Kuroki T, Nakatani S, et al. Randomised trial of effects of interferon-a on incidence of hepatocellular carcinoma in chronic active hepatitis $C$ with cirrhosis. Lancet 1995 ; 346 : 1051-5.

26. Fattovich G, Giustina G, Degos F, et al. Morbidity and mortality in compensated cirrhosis type C: a retrospective followup study of 384 patients. Gastroenterology 1997 ; 112 : 463-72

27. Yoshida $H$, Shiratori $y$, Moriyama $M$, et al. Interferon therapy reduces the risk for hepatocellular carcinoma : national surveillance program of cirrhotic and noncirrhotic patients with chronic hepatitis $C$ in Japan. Ann Intern Med 1999 ; 131 : 174-81.

28. Marcellin P. Hepatitis C: the clinical spectrum of the disease. J Hepatol 1999 ; 31 : (suppl 1) : 9-16.

29. Marcellin P, Lévy $S$, Erlinger S. Treatment of patients with chronic HCV infection with normal serum ALT levels. Hepatology 1997 ; 26 (suppl 1) : 133S-136S.

30. Poynard T. Quelles sont les indications du traitement dans l'hépatite aiguë C ? Gastroenterol Clin Biol 1997 ; 21 : S66-S69.

31. Jaeckel $\varepsilon$, Cornberg $M$, Wedemeyer $\mathrm{H}$, et al. Treatment of acute hepatitis $C$ with interferon alfa-2b. $N$ Engl J Med 2001 ; 345 : 1452-7.

32. Gervais A, Boyer N, Marcellin P. Co-infection VIH-VHC en I'an 2001. La lettre de l'infectiologue 2001 ; Tome XVI, N 6 : 186-90.

33. Causse X, Payen JL, Izopet J, Babany G, Saint Marc Girardin MF and the French Multicenter Study Group. Does HIV-infection influence the response of chronic hepatitis $C$ to interferon treatment? A French multicenter prospective study. J Hepatol 2000 ; 32: 1003-10.

34. Landau A, Batisse D, Duong Van Huyen JP, et al. Efficacy and safety of combination therapy with interferon-a $2 b$ and ribavirin for chronic hepatitis $\mathrm{C}$ in HIV infected patients. AIDS $2000 ; 14$ : 839-44.

35. Zylberberg H, Benhamou Y, Lagneaux JL, et al. Safety and efficacy of interferonribavirin combination therapy in HCV-HIV coinfected subjects : an 
early report. Gut 2000 ; 47 : 608-9.

36. Féray C, Caccamo L, Alexander GJM, et al. European collaborative study on factors influencing outcome after liver transplantation for hepatitis C.

Gastroenterology 1999 ; $117: 619-25$

37. Bizollon T, Palazzo U, Ducerf C, et al. Pilot study of the combination of alpha interferon and ribavirin as therapy of recurrent hepatitis $C$ after liver transplantation. Hepatology 1997 ; 26 : 500-504.

38. Martinot-Peignoux $M$, Roudot-Thoraval F, Mendel I, et al. Hepatitis C virus genotypes in France : relationship with epidemiology, pathogenicity and response to interferon therapy J Viral Hepatitis 1999 ; 6 : 435-43.

39. Martinot-Peignoux M, Boyer
$N$, Pouteau M, et al. Predictors of sustained response to alpha interferon therapy in chronic hepatitis C. J Hepatol $1998 ; 29: 214-23$.

40. Gervais A, Boyer B, Marcellin P. Tolerability of treatments for viral hepatitis. In Drug Safety. Ed. Adis international Ltd, Auckland, New Zealand, Vol. 24, N 5, 2001 pp375-384.

41. Asselah T, Martinot M, Boyer N, et al. Variabilité génétique du virus de I'hépatite $C$ : implications cliniques. Gastroenterol Clin Biol 2000 ; 24 : 175-84.

42. Pawlotsky JM, Pellerin M, Bouvier M, et al. Genetic complexity of the hypervariable region 1 (HVR 1) of hepatitis C virus. Influence on the characteristics of the infection and the response to alpha-interferon therapy in patients with chronic hepatitis C. J Med Virol

1998 ; 54 : 256-64.

43. Enomoto N, Sakuma I, Asahina $\mathrm{Y}$, et al. Mutations in the non structural protein $5 \mathrm{~A}$ gene and response to interferon in patients with chronic hepatitis $C$ virus $1 b$ infection. N Engl J Med 1996 ; $334: 77-81$.

44. Gervais $A$, Martinot $M$, Boyer $\mathrm{N}$, et al. Quantitation of hepatic hepatitis $\mathrm{C}$ virus RNA with chronic hepatitis C, relationship with severity of disease, viral genotype and response to treatment. J Hepatol 2001 ; 35 : 399-405.

45. Love RA, Parge HE, Wickersham JA, et al. The crystal structure of hepatits C virus NS3 proteinase reveals a trypsin-like fold and a structural zinc binding site Cell 1996; 87 : 331-42.

46. Yao N, Hesson T, Cable M, et al. Structure of the hepatitis C virus RNA helicase domain. Nat Struct Biol 1997 ; 4 : 463-7.

47. Von Weizsacker F, Wieland $S$, Kock J, et al. Gene therapy for chronic viral hepatitis : ribozymes antisense oligonucleotides, and dominant negative mutants. Hepatology 1997 ; $26: 251-5$

48. Abrignani $S$, Houghton $M$, Hsu HH. Perspective for a vaccine against hepatitis $C$ virus. J Hepatol 1999 ; 31 : 259-263.

49. Choo QL, Kuo G, Ralston R, et al. Vaccination of chimpanzees against infection by the hepatitis $C$ virus. Proc Natl Acad Sci USA $1994 ; 91$ : 1294-8.
TIRÉS À PART

P. Marcellin 